\title{
A COMPARATIVE STUDY OF THE UV LASER ABLATION OF VAN DER WAALS FILMS OF BENZENE DERIVATIVES
}

\author{
S. GEORGIOU, A. KOUBENAKIS,
} P. KONTOLETA and M. SYRROU

Foundation for Research and Technology-Hellas, Institute of Electronic Structure and Laser, P.O. Box 1527, 71110 Heraklion, Crete, Greece

\author{
(Received 10 May 1996)
}

\begin{abstract}
Ablation of thick $(\approx 15 \mu \mathrm{m})$ films of $\mathrm{C}_{6} \mathrm{H}_{6}, \mathrm{C}_{6} \mathrm{H}_{5} \mathrm{CH}_{3}$ and $\mathrm{C}_{6} \mathrm{H}_{5} \mathrm{Cl}$ at $248 \mathrm{~nm}$ and $193 \mathrm{~nm}$ is studied by means of time-of-flight quadrupole mass spectrometry. The dependence of the desorbate most probable translational energies on laser fluence is determined over the $\approx 20-200 \mathrm{~mJ} / \mathrm{cm}^{2}$ range. In all cases, the corresponding diagrams are found to exhibit "plateaus", in accord with the report by Braun and Hess [J. Chem. Phys. 99 (1993) 8330]. However, no specific correlation with the thermodynamic properties of the compounds is observed, thereby questioning the attribution of the "plateaus" to phase transformation of the films under ablation conditions. A high sensitivity of the distributions and intensities on the rate of deposition and the irradiation history of the films is observed, indicating the importance of the matrix "structure" for the distribution of the absorbed energy. On the other hand, the analysis of the total translational energies of the desorbates suggests that during ablation, efficient energy transfer occurs in the film. This possibility is further demonstrated by the observation of high translational energies and sputtering yields for $\mathrm{C}_{6} \mathrm{H}_{12}$ (nonabsorbing at $248 \mathrm{~nm}$ ) condensed in thickness of $\approx 1 \mu \mathrm{m}$ on top of $\mathrm{C}_{6} \mathrm{H}_{5} \mathrm{CH}_{3}$ films. These observations can be qualitatively explained in terms of the collisional sequence model. Alternatively, a photothermal model may be applicable under the provision that energy distribution in the films is limited due to imperfections introducing barriers (bottlenecks) to its 'flow'.
\end{abstract}

Keywords: Laser ablation; desorption; benzene derivatives; time-of-flight; energy transfer; cryogenic film

\section{INTRODUCTION}

Cryogenic films, that is films formed by the condensation of vapours of simple compounds on low-temperature substrates, present significant 
advantages for the detailed study of ablation processes. In particular, the physicochemical simplicity of the films ensures that the underlying mechanisms can be elucidated to a degree of detail that is usually not possible with technologically more important but highly complex substrates. Indeed, cryogenic films have proven most useful for examining the evolution from surface-mediated photochemistry to ablation [1-3], for investigating the nature of desorbate ejection under ablation conditions [4], for assessing desorption dynamics from binary mixtures [5], etc.

Recently, Braun and Hess employed $\mathrm{C}_{6} \mathrm{H}_{6}$ films as substrates for assessing in detail the ablation processes that are induced by pulsed IR irradiation [6c]. They were able to demonstrate that the desorbate most probable translational energy $\left(E_{\text {trans }}\right)$ does not increase monotonically with laser fluence $\left(F_{\text {LASER }}\right)$, but, instead it shows a "phase-transition"-like dependence. The $F_{\text {LASER }}$ range over which the characteristic "plateau" of the diagram appears corresponds to film temperatures that are suggestive of the solid-liquid transformation of the compound. In view of this result, ablation, at least in the IR spectral region, seems to be essentially photothermal in nature.

Hess and Braun suggested further that their observations may also hold for the UV-induced ablation of cryogenic films. However, a corresponding study in the UV was not performed. In fact, a recent examination [7] of the UV-ablation of several benzene derivatives failed to find any correspondence between the ablative thresholds and the thermodynamic properties of the compounds. Sputtering efficiencies were suggested to correlate, instead, with the photochemical activity of the studied compounds.

For examining the reasons for the discrepancy between the two studies, we studied previously [8b] the $248 \mathrm{~nm}$-induced ablation of solid solutions of $\mathrm{C}_{6} \mathrm{H}_{5} \mathrm{Cl}$-serving as the chromophore-in a nonabsorbing $\mathrm{C}_{6} \mathrm{H}_{12}$ host. Surprisingly, dilution of $\mathrm{C}_{6} \mathrm{H}_{5} \mathrm{Cl}$ in $\mathrm{C}_{6} \mathrm{H}_{12}$ was found to modify only its sputtering yields (as compared with the ablation of its neat films), whereas its $E_{\text {trans }}$ values, as well as their dependence on $F_{\text {LASER }}$, were nearly unaffected. Thus, translational distributions appear to be insensitive to the composition and, hence, to the distribution of the absorbed energy, in the irradiated films. This result seriously questions the suggestion about a phase transformation of the films under ablation conditions. On a more general level, even 
the applicability of simple photothermal models to the description of UV ablation of cryogenic films seems highly doubtful.

As a way of checking further the generality of our observations, we compare herein the ablation of $\mathrm{C}_{6} \mathrm{H}_{6}, \mathrm{C}_{6} \mathrm{H}_{5} \mathrm{CH}_{3}$ and $\mathrm{C}_{6} \mathrm{H}_{5} \mathrm{Cl}$ films at $248 \mathrm{~nm}$. The three compounds absorb comparably at $248 \mathrm{~nm}[7,9]$, and they are characterized by similar electronic structures, differing almost exclusively in their thermodynamic properties [10]. Thus, their comparative study enables an accurate assessment of the influence of these properties on the UV-ablation. Furthermore, we have examined the wavelength dependence of the processes by studying additionally the ablation of $\mathrm{C}_{6} \mathrm{H}_{6}$ films at $193 \mathrm{~nm}$.

We find the $E_{\text {trans }}$ vs. $F_{\text {LASER }}$ diagrams to exhibit, in all cases, "plateaus". However, no specific correspondence with the melting and boiling points of the compounds can be established. Most interestingly a pronounced dependence of the ablation observables on the mode of deposition and the irradiation "history" of the films is observed. This high sensitivity clearly indicates the importance of film "structure" for the distribution of the absorbed energy and corroborates our previous conclusion about efficient localization of the energy under ablation conditions. On the other hand, the examination of the total desorbate energies suggests that a very high percentage of the deposited energy is carried away by the ejecta, indicating the operation of efficient energy transfer mechanism(s) in the film during ablation. This possibility is further demonstrated in the study of layered films.

The results are discussed in terms of two simple models. A phenomenological explanation of the observations is provided by the so-called 'collisional sequence' model. Alternatively, we propose that the energy distribution in the film may be limited by the presence of imperfections, thereby resulting in the localization of the absorbed energy and the development of "hot spots". This mechanism can explain our observations and is also consistent with the physical properties of the films, as these have been established by matrix-isolation spectroscopy and other studies.

\section{EXPERIMENTAL}

The experimental setup is described in detail elsewhere [8a,b]. Briefly, experiments are performed on a vacuum system consisting of two 
differentially pumped chambers. In the first cell, a liquid $\mathrm{N}_{2}$ dewar supports a suprasil substrate for the deposition of the films.

Gases, introduced via a stainless needle (i.d. $=1 \mathrm{~mm}$, situated $\approx 2$ $\mathrm{cm}$ away from the substrate), are generally (except when otherwise noted) condensed at rates of $0.10-0.25 \mu \mathrm{m} / \mathrm{min}$ to form films approximately $15 \mu \mathrm{m}$ thick. The deposition rate has been deduced by monitoring the absorption of a weak laser beam $\left(248 \mathrm{~nm}, F_{\text {LASER }}\right.$ $\approx 0.4 \mathrm{~mJ} / \mathrm{cm}^{2}$ ) as a function of time. Alternatively, film growth is followed by monitoring the diffraction pattern formed by the interference of the refracted and incident $\mathrm{HeNe}$ beam on the substrate. In all cases, the vacuum chamber is pumped to a base pressure of $\approx 5 \times 10^{-8}$ mbar, before irradiation commences.

For the production of the layered films, $\mathrm{C}_{6} \mathrm{H}_{5} \mathrm{Cl}$ was first deposited as above and subsequently $\mathrm{C}_{6} \mathrm{H}_{12}$ was condensed for 2-5 min under well-controlled flow conditions. Under identical deposition conditions, $\mathrm{C}_{6} \mathrm{H}_{12}$ was found to form 1-2 $\mu \mathrm{m}$ thick films on suprasil. It is assumed that films of the same thickness are formed when condensed $\mathrm{C}_{6} \mathrm{H}_{5} \mathrm{Cl}$ serves as substrate instead. The analysis of the amount of $\mathrm{C}_{6} \mathrm{H}_{12}$ desorbing per pulse is consistent with this assumption.

The second chamber $\left(P<10^{-8}\right.$ mbar $)$ houses the mass spectrometer (Balzers QMG 412) which views the irradiated area in the plane defined by the irradiation axis and the normal to the substrate under a solid angle of $\approx 7 \times 10^{-5} \pi$ str. The distance between the substrate and the ion source of the mass spectrometer is $28 \mathrm{~cm}$. The signals are amplified on a preamplifier (Keithley: $\tau_{\text {rise }} \approx 15 \mu \mathrm{sec}$ ), recorded on a LeCroy LC9400 and sent to a computer. The operation of the mass spectrometer was calibrated according to the criteria established by Braun and Hess [11]. The operating conditions were chosen such as to have minimum transit times through the mass spectrometer, under the provision of retaining enough sensitivity. A low electron energy value (nominally, at $25 \mathrm{eV}$ ) was employed for minimizing cracking of the parent compound in the ion-source of the mass spectrometer.

Irradiation is effected with the output of a Lambda Physik EMG 150 excimer laser, operating either on $\mathrm{ArF}$ at $193 \mathrm{~nm}$ (nominal pulse duration of $17 \mathrm{nsec}$ ) or on $\mathrm{KrF}$ at 248 (nominal duration of $30 \mathrm{nsec}$ ). All experiments reported herein were performed at fixed incident and detection angles of the laser beam (approximately $75^{\circ}$ and $15^{\circ}$, respectively, 
with respect to the normal to the substrate). The beam is weakly focused to a rectangle of approximately $4 \times 10 \mathrm{~mm}^{2}$ along the substrate. Fluences are varied by adjusting the discharge voltage of the laser and by the use of filters (meshes). Variation in the laser fluence is $\approx 15 \%$. Light scattering at the film interface is determined from the intercept of the best linear fits to the transmission data recorded as a function of the film thickness (see above), i.e. we assume that the transmitted intensity is given by $I=I_{0} \mathrm{e}^{-\alpha x(t)}-I_{\text {scatt }}$, where $\alpha$ is the absorption coefficient and $x(t)$ the thickness of the film at time $t$. At $248 \mathrm{~nm}$, scattering losses for all compounds are measured to be $0.3 \pm 0.1$ of the incident beam. A calculation of the reflection losses on the basis of the refractive index $[12,13]$ of the compounds yields a value of 0.24 , in good correspondence with our determination. Losses at $193 \mathrm{~nm}$ have been taken into account based on the calculated [13] value of 0.45 .

Usually, 15-20 pulses are averaged per time-of-flight (TOF) curve, but at low $F_{\text {LASER }}$, as many as $40-80$ pulses are necessary for recording good-quality spectra. The TOF curves are corrected for the transit time through the mass spectrometer and for the inverse dependence of the ionization efficiency of the neutrals on their velocity. Most probable $\left(E_{\text {trans }}\right)$ kinetic energies of the species are determined from the $t_{\max }$ of the corrected curves.

Average and mean square speeds are calculated from the normalized flux distributions by multiplication by the appropriate factor (i.e., $L / t$ or $\left.(L / t)^{2}\right)$ in each case and integration over time. We have characterized the width of the translational distributions by means of the speed ratio, defined as [14]:

$$
\mathrm{SR}=(32 / 9 \pi-1)^{-1 / 2}\left(\left\langle v^{2}\right\rangle /\langle v\rangle^{2}-1\right)^{1 / 2}
$$

By way of example, the speed ratio for the desorbates detected in the ablation of $\mathrm{C}_{6} \mathrm{H}_{6}$ films at $248 \mathrm{~nm}$ is illustrated in Figure 1. At low fluences, the $\mathrm{SR}$ is larger than 1 (i.e., the distribution is broader than a Maxwell-Boltzmann one with the same $\left\langle v^{2}\right\rangle$ ), but rapidly decreases to values smaller than unity, suggestive of a rather "tight" expansion of the desorbates. Similar dependencies are also found for the other systems studied herein. Importantly, since the "width" of the distributions is fluence-dependent, there is no simple correlation between the 


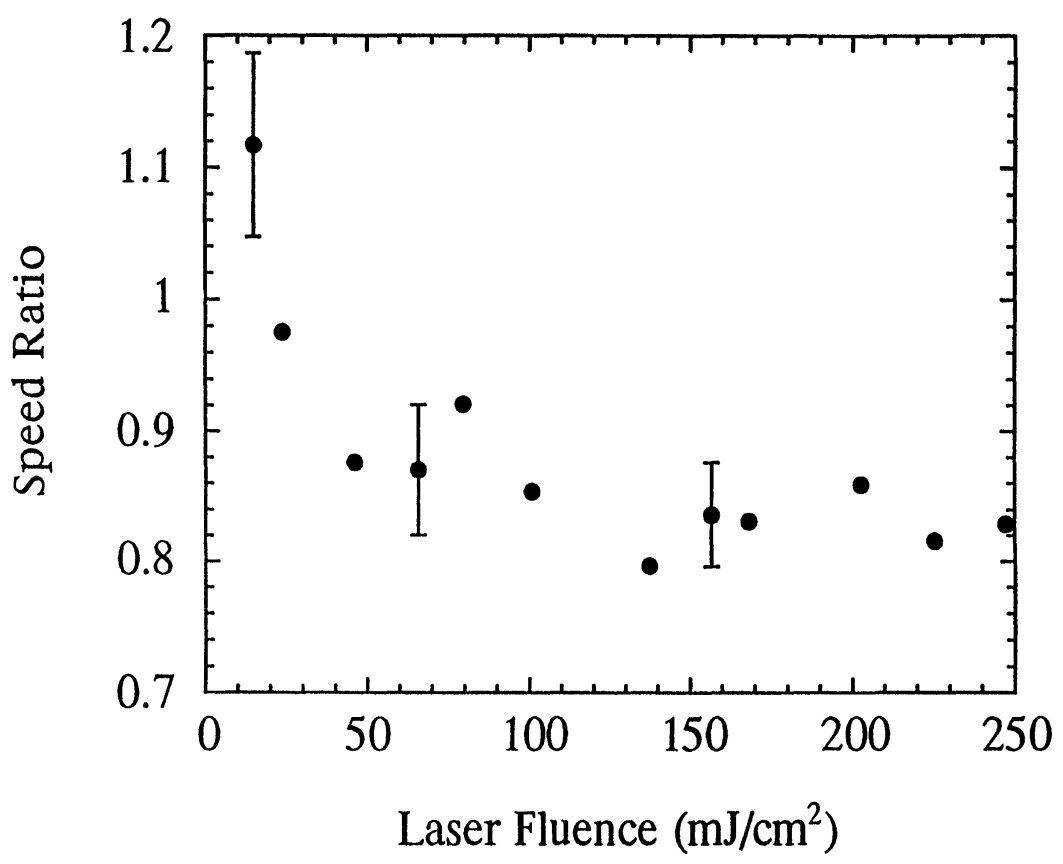

FIGURE 1 Speed ratios of the desorbate TOF distributions recorded in the ablation of $\mathrm{C}_{6} \mathrm{H}_{6}$ films at $248 \mathrm{~nm}$. The error bars represent $2 \sigma$, as determined from at least 6 different measurements of each datum point. Similar $F_{\mathrm{LASER}}$-dependences were also observed for the other systems examined herein. By comparison with Figures 2 and 3, it will be noticed that the determination of the speed ratios is subject to a rather large error. The reason for this seems to be the high sensitivity of the TOF shapes to the "structure" of the films.

most probable and average kinetic energies. Consequently, the two parameters differ somewhat in their dependences on $F_{\text {LASER }}$. In the following, we have opted to concentrate only on the $E_{\text {trans }}$ parameter, so that there is full correspondence with the study of Braun and Hess.

Intensities are determined by integration of the flux distributions over time. From these, the differential yield of desorbates (necessary for estimating the $E_{\text {TOTAL }}$ depicted in Fig. 6) is determined by accounting for the ionization and detection efficiencies of the compounds on the basis of their room-temperature mass spectra recorded at different pressures. (Pressure readings are corrected for the sensitivity of the ionization gauge). 
All compounds (Aldrich) are of high purity $(99.5 \%$ or better) and were subjected to careful trap-to-trap distillation and freeze-pumpthaw cycles.

\section{RESULTS AND DISCUSSION}

\section{a. F $_{\text {LASER }}-$ Dependence of the Desorbate Translational Energies}

The three compounds $\mathrm{C}_{6} \mathrm{H}_{6}, \mathrm{C}_{6} \mathrm{H}_{5} \mathrm{CH}_{3}$ and $\mathrm{C}_{6} \mathrm{H}_{5} \mathrm{Cl}$ absorb significantly at $248 \mathrm{~nm}$, with extinction coefficients (in the liquid phase) that range from $1900 \mathrm{~cm}^{-1}$ (the chloro species) to $2400 \mathrm{~cm}^{-1}$ (toluene [7b]). Accordingly, upon irradiation of their films, we detect desorption signals at fluences as low as $10 \mathrm{~mJ} / \mathrm{cm}^{2}$. For characterizing the process, we examine first the $F_{\text {LASER }}$-dependence of the most probable translational energies $\left(E_{\text {trans }}\right)$ of the detected desorbates (Fig. 2).

In accord with the suggestion by Braun and Hess, all graphs are found to exhibit a well-defined plateau at relatively low fluences, while a second one becomes evident at fluences higher than $\approx 180 \mathrm{~mJ} / \mathrm{cm}^{2}$. The comparison of the graphs does support, in some respects, the suggestion of a phase transformation. For instance, the $F_{\text {LASER }}$ ranges of the "plateaus" scale approximately as the latent heats of the compounds (corrected for the different absorption coefficients of the compounds). The same also holds with regard to the relative order of appearance of the plateaus.

On the other hand, we note some interesting incompatibilities with the phase-transformation concept. The fluences at which the "plateaus" first appear are somewhat lower than what is estimated as necessary for reaching the phase-transformation points (Tab. I). In particular, in the case of $\mathrm{C}_{6} \mathrm{H}_{5} \mathrm{Cl}$, the plateau appears at a $F_{\text {LASER }}$ value almost half of that expected from a simple photothermal model. It seems as if the absorbed energy is distributed over a smaller volume than what is presumed on the basis of the absorption coefficients of the compounds.

Interestingly, in the case of $\mathrm{C}_{6} \mathrm{H}_{6}$, the extinction coefficient at $248 \mathrm{~nm}$ turns out (fortuitously) to be approximately equal to that at the wavelength employed in the IR-study. Yet, there are some notable differences between Figure 2(a) herein and the corresponding graph in 


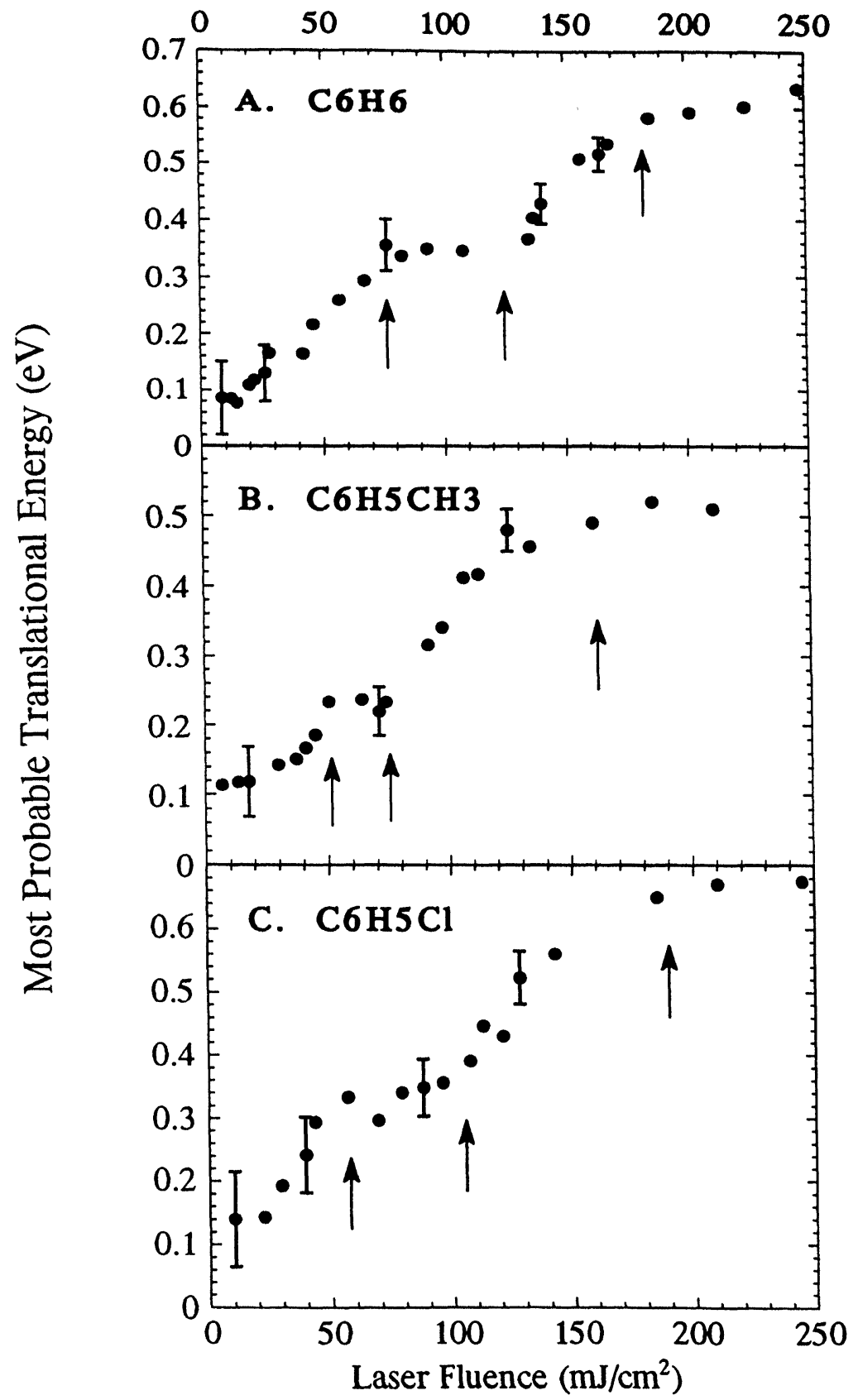

FIGURE $2 F_{\text {LASER }}$-dependence of the most probable $E_{\text {trans }}$ of the parent molecules detected in the ablation of thick $\mathrm{C}_{6} \mathrm{H}_{6}, \mathrm{C}_{6} \mathrm{H}_{5} \mathrm{CH}_{3}$ and $\mathrm{C}_{6} \mathrm{H}_{5} \mathrm{Cl}$ films at $248 \mathrm{~nm}$. In each case, the error bars represent $2 \sigma$, as determined from at least 6 different measurements of each datum point. The arrows indicate plateau regions. 
TABLE I Comparison of fluences at which phase transitions (melting and boiling points) of the films are expected to occur with the values at which the plateaus are actually observed

\begin{tabular}{lcc}
\hline Compound & $\begin{array}{c}\text { Calculated Fluence } \\
\left(\mathrm{mJ} / \mathrm{cm}^{2}\right)\end{array}$ & $\begin{array}{c}\text { Observed } \\
\text { Fluence } \\
\left(\mathrm{mJ} / \mathrm{cm}^{\mathrm{b}}\right)\end{array}$ \\
\hline $\mathrm{C}_{6} \mathrm{H}_{6}{ }^{\mathrm{c}}$ & 90 & 75 \\
& 142 & 140 \\
$\mathrm{C}_{6} \mathrm{H}_{5} \mathrm{CH}_{3}{ }^{\mathrm{d}}$ & 200 & 180 \\
& 57 & 50 \\
$\mathrm{C}_{6} \mathrm{H}_{5} \mathrm{Cl}^{\mathrm{e}}$ & 83 & 75 \\
& 265 & 170 \\
& 120 & 55 \\
& 170 & 120 \\
& 310 & 180 \\
\hline
\end{tabular}

${ }^{\text {a }}$ For each compound, the tabulated numbers represent the fluences for reaching the melting point, for "overcoming" it and finally for reaching the boiling point. Film temperatures are calculated on the basis [7] of $\Delta \mathrm{T}=\alpha F_{\mathrm{LASER}} /\left(C_{p}{ }^{*} \rho\right)$, where $\alpha$ is the extinction coefficient of the compound, $\rho$ its density (in $\mathrm{gr} / \mathrm{cm}^{3}$ ) and $C_{p}$ its heat capacity (in $\mathrm{J} / \mathrm{gr} \mathrm{K}$ ). All $C_{p}$ and $\Delta \mathrm{H}_{\text {fusion }}$ values are taken from ref. 15. The results of this simple formula were tested versus a more elaborate treatment taking account of the energy losses due to sublimation (as suggested by Vertes and Levine) [31-32] for the case of $\mathrm{C}_{6} \mathrm{H}_{5} \mathrm{Cl}$. Understandly, the simple formula underestimates the fluences at which the phasetransition points occur (especially the boiling point), but the discrepancy is rather small and does not justify the more elaborate treatment. ${ }^{b}$ The tabulated numbers represent, for each compound, the $F_{\text {LASER }}$ values between which the first $E_{\text {trans }}$-plateau is observed and the $F_{\text {LASER }}$ at which the second plateau is clearly attained.

${ }^{\mathrm{c}} \rho_{\text {liquid }}=0.89 \mathrm{gr} / \mathrm{cm}^{3}, \rho_{\text {solid }}=1.01 \mathrm{gr} / \mathrm{cm}^{3}, \Delta \mathrm{H}_{\text {fusion }}=9.86 \mathrm{~kJ} / \mathrm{mole}$, $c_{p, \text { solid }}=14.23+0.37 \mathrm{~T} \mathrm{Jmole}{ }^{-1} \mathrm{~K}^{-1}$ (linear fitting to the reported in the literature values), $c_{p, \text { liquid }}=1.52-1.3 \times 10^{-3} \mathrm{~T}+6.9 \times 10^{-6} \mathrm{~T}^{2}$ $\mathrm{Jgr}^{-1} \mathrm{~K}^{-1}$ (valid over the $280 \mathrm{~K}-680 \mathrm{~K}$ range). [15]

${ }^{\mathrm{d}} \rho_{\text {liquid }}=0.87 \mathrm{gr} / \mathrm{cm}^{3}, \Delta \mathrm{H}_{\text {fusion }}=6.64 \mathrm{~kJ} / \mathrm{mole} ; c_{p}=187.44-0.73 \mathrm{~T}+$ $2.96 \times 10^{-3} \mathrm{~T}^{2} \mathrm{Jmole}^{-1} \mathrm{~K}^{-1}$ (valid over the $165 \mathrm{~K}-312 \mathrm{~K}$ range). [15] ${ }^{\mathrm{e}} \rho_{\text {liquid }}=1.11 \mathrm{gr} / \mathrm{cm}^{3}, \quad c_{p}=150 \mathrm{~J} \mathrm{~mole}^{-1} \mathrm{~K}^{-1}$ (mean value over $90-320 \mathrm{~K}$ range); $\Delta \mathrm{H}_{\text {fusion }}=9.56 \mathrm{~kJ} \mathrm{~mole}^{-1}$.

that study. For instance, the determined $E_{\text {trans }}$ values are much higher than those in the IR experiment. Most importantly, the second "plateau" at high $F_{\text {LASER }}$ was not observed previously. This additional plateau certainly does not derive from gas-dynamic postdesorption effects (i.e., modifications to the desorbate translational distributions). This is clearly demonstrated by the fact that the corresponding desorption intensities 
(Fig. 4), as well as the speed ratio of the distributions (Fig. 1), have levelled off by the time the $E_{\text {trans }}$ plateau is reached. Thus, it is tempting to attribute the appearance of this plateau to the contribution of film process(es) beyond those operative in the IR ablation. However, there is presently no information in support of this. At any rate, the observed differences not only question a simple thermal interpretation of the observed $E_{\text {trans }}$ vs. $F_{\text {LASER }}$ dependences, but also provide circumstantial evidence that UV ablation may not be equivalent to the IR one. ${ }^{\# 1}$

As a further test of the phase-transformation concept, we have examined the wavelength dependence of the translational parameters of the desorbates. To this end, Figure 3 depicts the $E_{\text {trans }}$ vs. $F_{\text {LASER }}$ diagram determined in the ablation of $\mathrm{C}_{6} \mathrm{H}_{6}$ films at $193 \mathrm{~nm}$. Absorption at this wavelength is $\approx 100$ times more intense than that at $248 \mathrm{~nm}[16,17]$. Consequently, the melting and boiling points of the

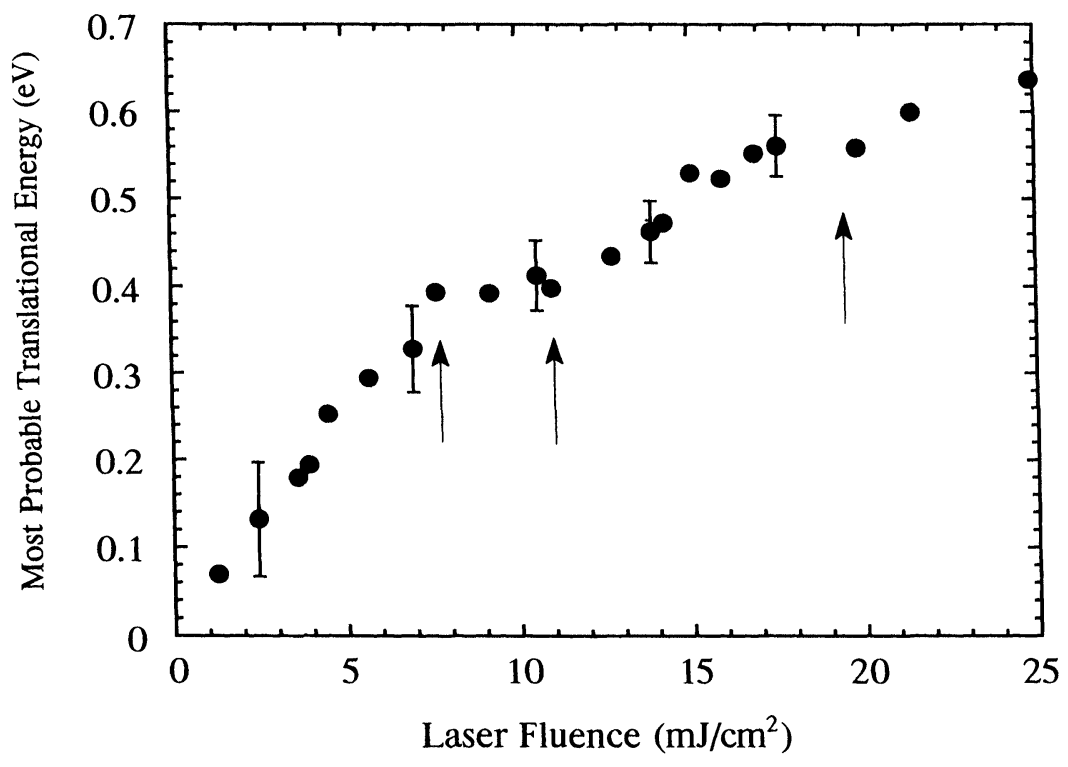

FIGURE $3 F_{\text {LASER }}$-dependence of the most probable $E_{\text {trans }}$ of the parent molecules detected in the ablation of thick $\mathrm{C}_{6} \mathrm{H}_{6}$ films at $193 \mathrm{~nm}$. The error bars represent $2 \sigma$, as determined from 5 different measurements. The arrows indicate plateau regions.

\footnotetext{
\#1 It must be noted, however, that differences in the experimental conditions of the two studies, and in particular, in the pusle widths of the employed lasers, could be partly responsible for the observed differences in the results.
} 

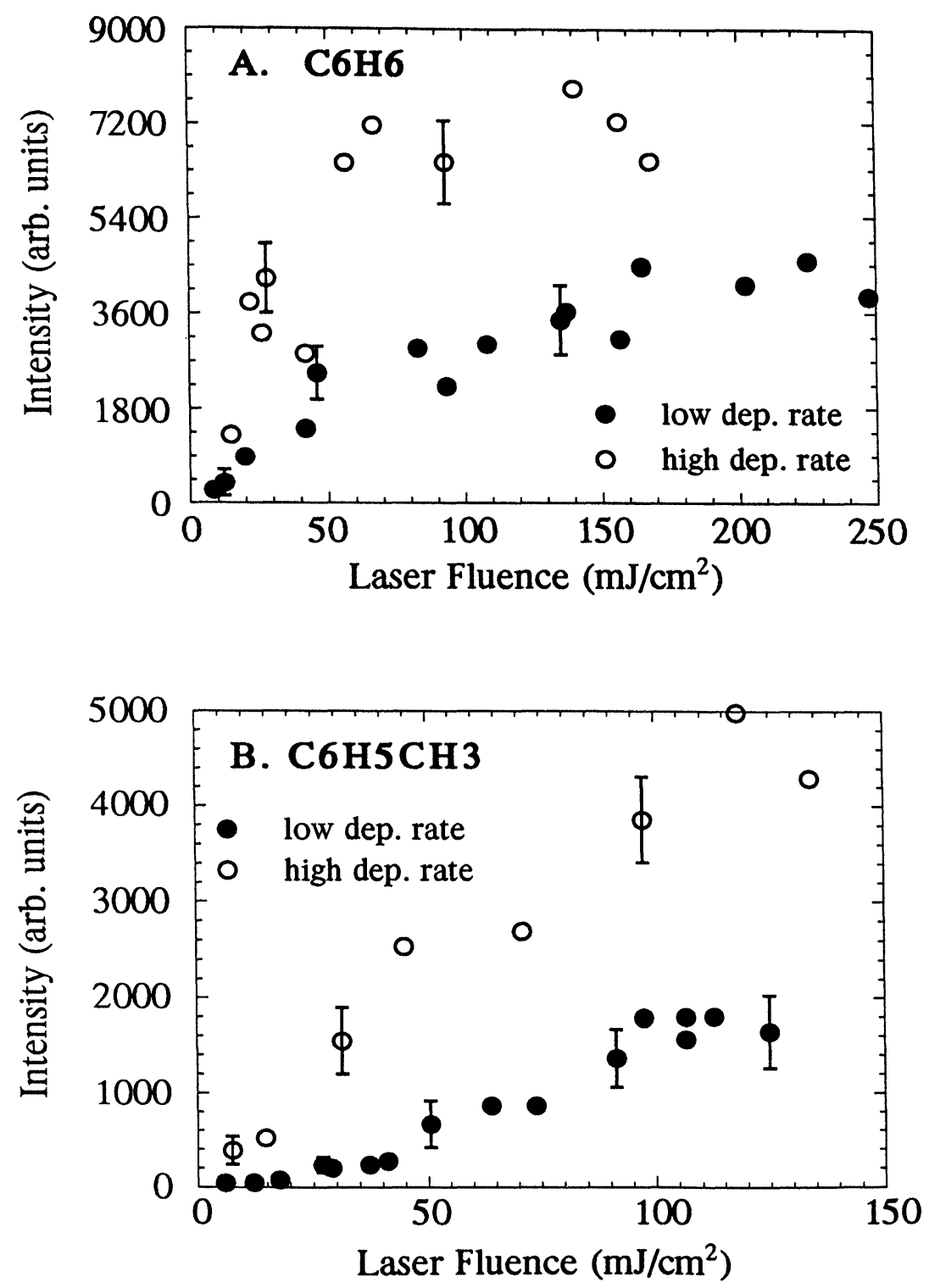

FIGURE 4 Plot of the intensities of desorbates from films deposited at rates of $\approx 1-2 \mu \mathrm{m} / \mathrm{min}$ (open circles) and $\approx 0.1-0.2 \mu \mathrm{m} / \mathrm{min}$ (filled circles). According to the information available from matrix-isolation studies, we tentatively consider that the films deposited in the first case to be highly amorphous and porous. 
compound should be attained at correspondingly lower fluences. Indeed, the plateaus appear at lower fluences, but in sharp contrast with the prediction, the decrease is hardly a factor of 10 . Evidently, the discrepancy is significant and cannot be accounted for by inaccuracies in the estimation of the absorbed energies or fluences. In particular, the wavelength dependence of the refractive index of the solid is known very accurately [17]. On the basis of the available information, we can be confident that light scattering losses at the two wavelengths have been accurately taken into account. Thus, once more, we find that the $E_{\text {trans }}$ vs. $F_{\text {LASER }}$ features do not scale as the latent heats of the compound or, alternatively, the depth of absorption in the film is not correctly described by the literature extinction coefficients. In all, the comparative study does not provide any clear support for the suggestion of phase transformation of the films.

On the other hand, we note a surprisingly good correspondence with the study by Tsuboi et al. [7] concerning the $248 \mathrm{~nm}$ ablation of the compounds examined herein in the liquid phase. By using a photoacoustic technique, they determined the thresholds for plume ejection from room-temperature samples of $\mathrm{C}_{6} \mathrm{H}_{6}, \mathrm{C}_{6} \mathrm{H}_{5} \mathrm{CH}_{3}$ and $\mathrm{C}_{6} \mathrm{H}_{5} \mathrm{Cl}$ at $100 \mathrm{~mJ} / \mathrm{cm}^{2}, 35 \mathrm{~mJ} / \mathrm{cm}^{2}$ and $60 \mathrm{~mJ} / \mathrm{cm}^{2}$. ${ }^{2}$ All three thresholds are in good accord with the $F_{\text {LASER }}$ values at which the first "plateau" appears in the graphs in Figure 2. In view of the good agreement, a correlation between the photoacoustic measurements and the time-offlight ones seems justifiable, though evidently its validity must be verified in a wider set of compounds. To the extent that it holds, the correlation suggests the threshold of ablation to be approximately

${ }^{22}$ Tsuboi et al. have suggested [7] that the ablation thresholds for the different compounds scale as their photochemical activity. Indeed, as described elsewhere, we have observed [8a] efficient photodissociation and reactivity of the fragments in the ablation of films of $\mathrm{C}_{6} \mathrm{H}_{5} \mathrm{Cl}$ and of other compounds $\left(\mathrm{CH}_{3} \mathrm{I}, \mathrm{CH}_{2} \mathrm{Cl}_{2}\right.$ etc). However, it is not clear if there is any correspondence between the observed reactivity patterns and the ablation thresholds. In particular, the fact that the $E_{\text {trans }}$ vs. $F_{\text {LASER }}$ graph in the ablation of $\mathrm{C}_{6} \mathrm{H}_{5} \mathrm{Cl} / \mathrm{C}_{6} \mathrm{H}_{12}$ is nearly identical to the one determined [8b] for neat $\mathrm{C}_{6} \mathrm{H}_{5} \mathrm{Cl}$ seems to argue against the photochemical mechanism (since in the mixture the degree of photolysis may be decreased by the amount of dilution of the chromophore). Plausibly, the quantum yield of dissociation of $\mathrm{C}_{6} \mathrm{H}_{5} \mathrm{Cl}$ in $\mathrm{C}_{6} \mathrm{H}_{12}$ solvent is much higher than that in the neat compound, thereby compensating for the smaller concentration of the compound in the mixture. Unfortunately, there is not sufficient information on the photochemistry of $\mathrm{C}_{6} \mathrm{H}_{5} \mathrm{Cl}$ to enable a concrete conclusion to be reached. 
independent of the initial temperature and phase (liquid or solid) of the sample. This is quite surprising, but may perhaps be justified by the fact that the structure of the amorphous solids resembles closely that of the liquids [18-20]. At any rate, according to the suggested correlation, the features in $E_{\text {trans }}$ vs. $F_{\text {LASER }}$ diagrams appear to be indicative of distinct stages in the ablation process; however, the nature of the factors responsible for these features cannot be simple phase transformations of the films. At the very least, additional factors must be involved.

\section{b. Dependence of Ablation Observables on Film "Structure"}

A particular interesting observation that further argues against the possibility of a simple phase-transformation concerns the unexpected sensitivity of the ablation observables on the mode of deposition of the films and their irradiation "history". The importance of these experimental parameters for the desorption efficiency is exemplified in Figure 4 and 5. The former depicts the effect of the deposition rate on the desorbate intensities, while the latter depicts the evolution in intensities observed with continuing irradiation of the films. ${ }^{\# 3}$ In the experiments of Figure 6, the laser repetition rate was $0.5-1 \mathrm{~Hz}$ (in fact, in many cases, the delay between recording of successive data points was much longer, in the range of minutes). Evidently, these effects survive for long times, which conclusively demonstrates that the responsible mechanism is of a structural nature (i.e., entailing modifications to the "film" structure). Electronic or thermal factors can be confidently ruled out.

We have tried to establish the nature of the structural modifications, involved via fluorescence monitoring of the films; however, our preliminary attempts have thus far proven unsuccessful. Nevertheless, as discussed below, there is much evidence from matrix-isolation studies [21-23] that the main effect of the deposition rate concerns the degree of porosity and irregularity of the produced films. In particular, the higher the deposition rate, the higher the degree of porosity of the

${ }^{\# 3}$ These effects are paralleled by corresponding changes in the $F_{\mathrm{LASER}}$-graph of the $E_{\text {trans }}$. However, the change in the $E_{\text {trans }}$ may be a side effect of the enhanced postdesorption collisional dynamcis that occurs as a result of the increased quantity of ejected material. 


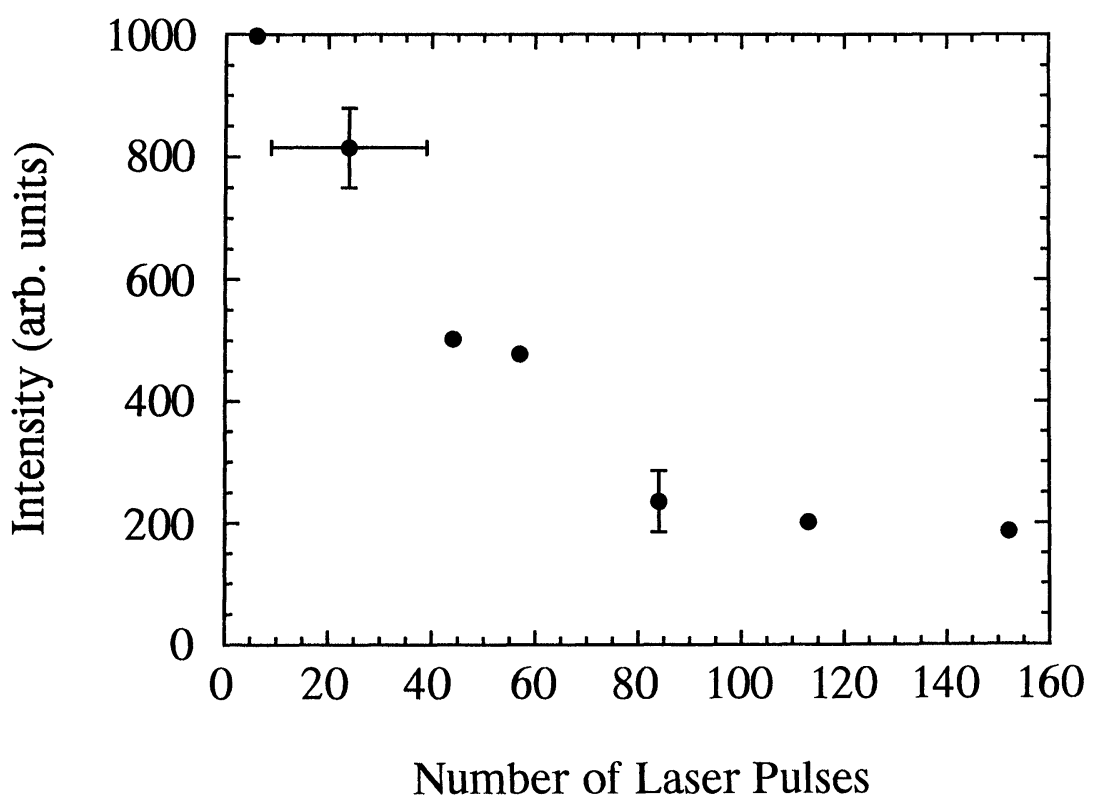

FIGURE 5 The figure illustrates the decrease in desorbate intensities that is observed with continuing irradiation of initially "amorphous" films (films deposited at high rates). In this case $\lambda=193 \mathrm{~nm}\left(F_{\text {LASER }} \approx 10 \mathrm{~mJ} / \mathrm{cm}^{2}\right)$, but similar effects are also observed for irradiation at $248 \mathrm{~nm}$. It is noted that the effects illustrated in this Figure are very sensitive to the mode of film deposition. Empirically, we have found that deposition at $\approx 0.1-0.4 \mu \mathrm{m} / \mathrm{min}$ results in the "best" films, where the effects of laser-induced annealing are the least pronounced. In that case, signal variations in the initial 300-500 pulses are relatively small.

films. Under this assumption, the implication of Figure 4 is that sputtering efficiencies are much enhanced from amorphous matrices as compared with well-deposited ones. Similarly, the effect of continuing irradiation in Figure 5 must derive from the attentant laser-induced annealing of the matrix. Tentatively, we suggest that in the initially deposited films, the highly grainy structure of the films results in efficient localization of the absorbed energy. As, however, irradiation proceeds, the film is annealed with a corresponding relaxation of the constraints that limit the distribution of the energy. This, then, would explain the dramatic decrease in desorption efficiency observed in Figure 5. At any rate, independently of the specific explanation, the results indicate that the energy distribution during ablation is highly sensitive to the "structure" of the films. 


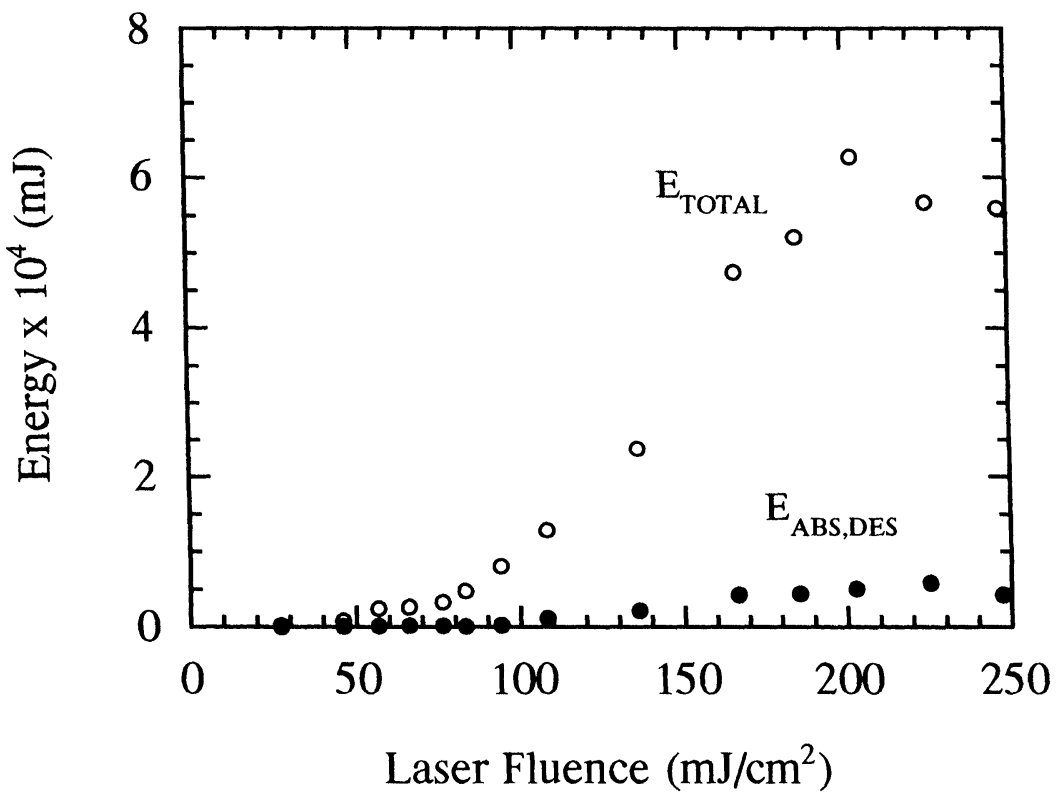

FIGURE 6 The figure depicts the $E_{\mathrm{TOTAL}}$ and $E_{\mathrm{ABS} \text {, Des }}$ as function of the incident $F_{\text {LASER }}$, in the ablation of $\mathrm{C}_{6} \mathrm{H}_{6}$ films at $248 \mathrm{~nm}$. $E_{\text {TOTAL }}$ represents the total translational energy of the detected desorbates. (i.e., those desorbates ejected in the solid angle subtended by the mass spectrometer), while $E_{\mathrm{ABS} \text {, DES }}$ represents the energy absorbed by the detected desorbates themselves, when in the film.

Interestingly, a similarly pronounced influence of the substrate "structure" on desorption has also been reported [24] in Matrix-Assisted Laser Desorption (MALD) studies. In fact, some observations closely resemble the results described above (Fig. 5). Again therein, the evidence suggests that the formation of different "crystalline domains" in the matrix affects the efficiency of desorption. The authors suggested that at a given fluence only crystallites up to a given size exceed the ablation threshold and sublime. This can happen if energy transfer among different "crystalline domains" of the samples is, as suggested above, hindered.

\section{c. Demonstration of Energy Transfer in the Ablation of Layered Films}

In section $a$, we considered the dependence of the $E_{\text {trans }}$ of the desorbates on $F_{\text {LASER }}$, with complete neglect of the desorption yields. For the consideration of both observables, we examine the total translational energy of the detected desorbates, $E_{\text {TOTAL }}=N_{\text {det }} \times\left\langle E_{\text {trans }}\right\rangle$, 
where $N_{\text {det }}$ represents the detected number of molecules and $\left\langle E_{\text {trans }}\right\rangle$ their average energies (Fig. 6). For all three compounds, the efficiency of energy disposal on the desorbates increases sharply with $F_{\text {LASER }}$, until becoming "saturated" at higher fluences. The levelling off reflects the fact that both the $\left\langle E_{\text {trans }}\right\rangle$ and the sputtering yields, as well, reach a "plateau" at these fluences. Saturation of desorption yields was also noted in the IR experiments. Braun and Hess suggested [6c] that the "saturation" results from the operation of "cage" effects that prevent material ejection beyond a specific depth. Our previous work [8a, 8b] fully supports their suggestion.

Most interestingly, the values of $E_{\text {ТОТАL }}$ indicated by Figure 6 are very high, especially if one considers that the observed species constitute only a small fraction of the ejected particles. In fact, only $\approx 5-10 \%$ of the carried energy is calculated on the basis of Beer's law to have been actually absorbed by the detected desorbates themselves (when in the film). As has already been discussed by Leone et al. [4a] and by Braun and Hess [6c], the results suggest a highly efficient energy transfer mechanism in the film under ablation conditions.

Considering the importance of this finding, we need a more direct approach for its demonstration and study. To this end, we deposited $\mathrm{C}_{6} \mathrm{H}_{12}$ in thickness of $\approx 1-2 \mu \mathrm{m}$ upon films of $\mathrm{C}_{6} \mathrm{H}_{5} \mathrm{CH}_{3}$ or of $\mathrm{C}_{6} \mathrm{H}_{5} \mathrm{Cl} . \mathrm{C}_{6} \mathrm{H}_{12}$ absorbs negligibly at $248 \mathrm{~nm}\left(\varepsilon_{\text {liquid }} \approx 0.02 \mathrm{~cm}^{-1}\right)$ and as indicated in Table II, the irradiation of its pure (neat) films results in very weak signals at $F_{\text {LASER }}$ lower than $\approx 220 \mathrm{~mJ} / \mathrm{cm}^{2}$. Nevertheless, when condensed on top of the absorbing films, $\mathrm{C}_{6} \mathrm{H}_{12}$ is found to desorb massively and with very high kinetic energies (Tab. II).

Most importantly, for the thicker $\mathrm{C}_{6} \mathrm{H}_{12}$ films (estimated thickness of $\approx 2 \mu \mathrm{m}$ ) that clearly exhibited the influence of the chromophore substrate, $\mathrm{C}_{6} \mathrm{H}_{5} \mathrm{CH}_{3}$ desorption is negligible $\left(\mathrm{I}_{\mathrm{C}_{6} \mathrm{H}_{5} \mathrm{CH}_{3}}<5 \%\right.$ of the $\mathrm{C}_{6} \mathrm{H}_{12}$ signal). The observation establishes ${ }^{\# 4}$ that the ejection of

${ }^{\# 4}$ Presumably, $\mathrm{C}_{6} \mathrm{H}_{5} \mathrm{CH}_{3}$ could still be desorbing but scattered off the detection angle. Though we cannot discount this possibility, the fact that $\mathrm{C}_{6} \mathrm{H}_{5} \mathrm{CH}_{3}$ and $\mathrm{C}_{6} \mathrm{H}_{12}$ have nearly equal molecular masses suggests that the two species should desorb with comparable angular distributions. Thus, the observation that the $\mathrm{C}_{6} \mathrm{H}_{5} \mathrm{CH}_{3}$ intensity is much smaller than that of $\mathrm{C}_{6} \mathrm{H}_{12}$ is expected to hold at all angles. At any rate, even if some $\mathrm{C}_{6} \mathrm{H}_{5} \mathrm{CH}_{3}$ does desorb, the validity of our conclusion will not be compromised. 
TABLE II Comparison of $\mathrm{C}_{6} \mathrm{H}_{12}$ desorption from thin film (deposited on top of thick absorbing matrix with that from its neat films $(\lambda=248 \mathrm{~nm})$

\begin{tabular}{lcccc}
\hline $\begin{array}{l}\text { FLUENCE } \\
\left(\mathrm{mJ} / \mathrm{cm}^{2}\right)\end{array}$ & $\mathrm{C}_{6} \mathrm{H}_{12}$ on $\mathrm{C}_{6} \mathrm{H}_{5} \mathrm{CH}_{3}$ substrate & \multicolumn{2}{c}{ neat $\mathrm{C}_{6} \mathrm{H}_{12}$ film $^{\mathrm{b}}$} \\
\hline & $\begin{array}{c}E_{\text {trans }} \\
\text { (in eV) }\end{array}$ & $\begin{array}{c}\text { Intensity } \\
\text { (arb. units) }\end{array}$ & $\begin{array}{c}E_{\text {trans }} \\
\text { (in eV) }\end{array}$ & $\begin{array}{c}\text { Intensity } \\
\text { (arb. units) }\end{array}$ \\
\hline 20 & 0.06 & 60 & $-^{\mathrm{c}}$ & - \\
75 & 0.13 & 600 & 0.12 & 52.3 \\
115 & 0.15 & 900 & 0.14 & 190 \\
215 & 0.22 & 2000 & 0.2 & 300 \\
\hline
\end{tabular}

${ }^{\text {a }}$ The reported numbers refer to time-of-flight spectra recorded in the first 5-10 pulses of irradiating $\approx 1.0 \mu \mathrm{m}$ thick $\mathrm{C}_{6} \mathrm{H}_{12}$ films on the indicated substrate. The variation in the determined parameters is of the order of $\approx 40 \%$.

${ }^{b}$ As described elsewhere [8c], the signals in the irradiation of neat $\mathrm{C}_{6} \mathrm{H}_{12}$ films exhibit a pronounced induction behavior. The numbers reported herein represent the finally attained $E_{\text {trans }}$ and intensity values. Thus, the comparison underestimates the effect of the underlying chormophore, since in this case, the reported intensity represents signal observed in the first 10 pulses, whereas the "induction" signal is recorded after usually $\approx 200$ pulses (at low $F_{\text {LASER }}$ ) and in $\approx 40$ pulses at the higher ones.

${ }^{\mathrm{N}}$ No signal observed in $\approx 250-300$ pulses.

$\mathrm{C}_{6} \mathrm{H}_{12}$ is not due to its entrainment into the plume of the chromophore. Rather, ejection occurs by energy that is somehow "transferred" from the underlying $\mathrm{C}_{6} \mathrm{H}_{5} \mathrm{CH}_{3}$ film to the desorbates.

Evidently, the ejection cannot be described as a typical evaporation or sublimation process; rather the concept of "explosive" phenomenon (i.e., the term initially coined by Chuang and Domen [1] for describing ablation from films) seems to be a much more fitting description. The experiment strongly indicates that during irradiation, "hot spots" are produced that induce ejection of the overlying material by essentially "mechanical" means (as by shock or "pressure" waves, etc). Evidently, the deposition of $\mathrm{C}_{6} \mathrm{H}_{12}$ on top of the absorbing compound serves to limit the relaxation of these "hot spots", thereby making their effect more pronounced than that in the ablation of the neat films. In this sense, the results of the layered experiments corroborate the tentative conclusion reached in the previous section that localization of the absorbed energy results in more efficient sputtering. 


\section{d. Theoretical Considerations}

Two different mechanisms seem at this point to be the simple to account for these observations. The first one is based on the concept of "collisional" sequences, introduced some time ago by Thomson [25] and Sigmund [26] and examined in detail by Johnson and coworkers [27]. According to this model, excitation results eventually in translationally "hot" species produced by the dissociation of the primary excited excimers (dimers). The "hot" species collide with their neighbours, thereby inducing collisional cascades by which their energy is transferred to the surface atoms. Ejection occurs when the energy reaching them exceeds their binding strength to the crystal. As discussed in detail elsewhere [8b], this model can qualitatively account for most observations made in the study of the UV-ablation of cryogenic films. Specifically, since the relative arrangement of the molecules in the matrix will affect the efficiency of energy transfer in the collisional sequences, the model easily accounts for the high sensitivity of the ablation observables to the "structure" of the films. Additionally, the efficiency of transfer should drop with increasing number of intervening collisions, thereby accounting for the fact that desorption is limited to a specifc depth (i.e., the cage effects suggested by Braun and Hess).

Yet, despite its phenomenological success, the model is rather simplistic. There is no doubt that the distribution of energy should be significant on nsec time scales. For instance, vibrational decay in these films is measured to be of the order of picoseconds [28-29]. Similarly, in the study of photoreactivity, product analysis is often consistent with thermalization of the nascent fragments on a psec time scale [30 ]. Overall, thermal relaxation during the irradiation of the films is expected to be significant, but the model of collisional sequences completely neglects this possibility. In that sense, the model fails to provide insight about the physical basis of the ablation mechanisms.

A way of rationalising the expectation of thermal relaxation with the experimental results on the UV ablation of cryogenic films seems to be afforded by the idea of "bottlenecks" introduced by Vertes and Levine [31-32]. According to these authors, matrix-assisted desorption of biomolecules (MALD) can be described by a photothermal model in which the energy absorbed by the host is freely distributed in the crystal lattice, but with its "flow" to the guest molecules being limited by 
frequency mismatches. The model has been successful in accounting for the fact that in MALD, highly labile biopolymers desorb with minimal degradation, despite the rather high laser fluences employed [33]. Evidently in the films we have been studying, such "bottlenecks" can not exist (frequencies being identical or nearly so); nevertheless, energy diffusion may be limited by the imperfections, voids etc., present in the deposited matrices.

Matrix-isolation spectroscopic studies [20-23,34] have yielded extensive evidence that vapour-deposited films are far from well-ordered, layered structures. Additionally, studies employing specific characterization techniques [35] (such as $\mathrm{x}$-ray powder diffraction and $\mathrm{H}_{2}$ absorption measurements) have demonstrated that the matrices have unexpectedly high surface areas, evidently because of the existence of large vacancies, voids and other imperfections. These experimental results have been given further support by molecular dynamics simulations [21].

In matrix-isolation studies, annealing techniques are often used in an effort to improve sample crystallinity, thereby enabling meaningful spectroscopic examination of the guest molecules [20-23]. However, in the case of our TOF experiments, no previous annealing is performed. Consequently, the examined films retain the high degree of porosity and irregularity created during their deposition.

The presence of imperfections will certainly have an effect on the distribution of the energy in the irradiated films. It is reasonable to suggest that energy transfer across the imperfections is hindered, thereby introducing "bottlenecks" analogous to those considered by Levine and Vertes. In that case, energy remains localized on the grains that have actually absorbed a photon. Because of their small size, the grains will achieve very high temperatures, with their resultant intense vaporization providing the driving force for the ejection of the overlying material, very much like in the pressure pulse model suggested by Johnson and Sundqvist [36-37].

Qualitatively at least, the model can account for most observations made in the UV ablation of van der Waals films. First of all, this model can explain the very high sensitivity of desorption efficiencies to the "structure" of the films. In highly amorphous films, grains should be, on average, of smaller size. Therefore, energy localization is correspondingly enhanced, thus resulting in higher local temperatures and "explosive force". As a result, signals should be correspondingly 
increased, as indeed observed. Second, the proposed model can explain the observed "energy transfer". If the relaxation of these "hot spots" is prevented, they will reach even higher pressures and effective "pushing force", exactly as demonstrated in the layered experiment. In that sense, the energy transfer mechanism under ablation conditions differs essentially from the electronic $E->T$ or $E->V$ ones suggested [38] in the surface-mediated, low fluence regime. The overlayer experiment demonstrates further that the ejection of the overlying material can take place without the "hot spot" itself being ejected. Plausibly, the vaporized molecules of the spot are backscattered into the film as a result of their collisions with the upper layers. The efficiency of backscattering should depend on the thickness and compactness of the overlying film. Thus, for "hot spots" in deeper layers, their material will not be able to "diffuse" through to the gas-phase, though evidently their relaxation can contribute to the ejection of the higher layers. This, then, accounts for the "cage effects" observed by Braun and Hess. Furthermore, the contribution of grains may explain why the "plateaus" in the $E_{\text {trans }}$ vs. $F_{\text {LASER }}$ graphs appear at lower $F_{\text {LASER }}$ than expected on the basis of the thermodynamic properties of the compounds. Finally, formation of "hot spots" is strongly indicated by the observation of "induction" and "memory" effects in the irradiation of weakly absorbing films (specifically, of $\mathrm{C}_{6} \mathrm{H}_{12}$ and $\mathrm{CH}_{2} \mathrm{Cl}_{2}$ ). These effects are discussed elsewhere [8c].

Based on this description, we suggest that the various stages in the $E_{\text {trans }}$ vs. $F_{\text {LASER }}$ diagrams illustrated in Figures 3 and 4 just represent different phases in the evolution of the formation and relaxation of the "hot spots" in the irradiated films. As for the $F_{\text {LASER }}$-ranges and the wavelength dependence of the "plateaus", these may be determined by the balance between the contributions of grains of different sizes (i.e., small grains reaching higher temperatures "competing" with the higher absorption probability of the larger ones).

A particularly important implication of the proposed mechanism is that most ejected material does not necessarily reach the high temperatures suggested by the photothermal model and may retain, to a large degree, its integrity. Hence, any photochemical processes that occur during the laser pulse will be subject to the strong constraints characteristic of solid phase (i.e., efficient geminate recombination of photoproduced radicals) $[38-40]$. We believe this to be the reason for the very inefficient 
photolysis observed in the ablation of van-der-Waals films. This possibility was indicated early on in the pioneering experiments of Leone and coworkers [4], and has been recently studied in detail in the ablation of films of $\mathrm{C}_{6} \mathrm{H}_{5} \mathrm{Cl}[8 \mathrm{a}]$ and others $\left(\mathrm{CH}_{2} \mathrm{Cl}_{2}\right.$ and $\left.\mathrm{CH}_{3} \mathrm{I}\right)$.

It is noted that the idea of vaporization in "hot spots" providing the driving force for the supersonic-like expulsion of material has already been advanced in somewhat different forms by Braun and Hess [6c], and by Campos et al. [5] in the study of binary $\mathrm{Xe} / \mathrm{Cl}_{2}$ films. Our description complements these suggestions by emphasizing the "structural" origins of the phenomenon. In particular, the discussion about the importance of the grainy structure of the films provides a possible rationalization for the formation of "hot" spots in what at first would seem to be uniform, homogeneous substrates (like the $\mathrm{C}_{6} \mathrm{H}_{6}$ films).

Of course, this is a highly qualitative and tentative picture. Further experiments are underway for its validation. In particular, the comparativestudy of amorphous vs. well-annealed films (by thermal means) seems to be a most promising approach for elucidating factors controlling energy distribution in the films under ablation conditions.

\section{SUMMARY}

Ablation of thick $\mathrm{C}_{6} \mathrm{H}_{6}, \mathrm{C}_{6} \mathrm{H}_{5} \mathrm{CH}_{3}$ and $\mathrm{C}_{6} \mathrm{H}_{5} \mathrm{Cl}$ films at $248 \mathrm{~nm}$ and $193 \mathrm{~nm}$ is addressed by monitoring the $F_{\text {LASER }}$-dependence of the desorbate $E_{\text {trans }}$, intensities and $E_{\text {TOTAL }}$. In accord with the suggestion by Braun and Hess, we find that "plateaus" are always observed in the $E_{\text {trans }}$ vs. $F_{\text {LASER }}$ diagrams. However, these plateaus do not exhibit any specific correlation with the thermodynamic properties of the compounds, so that their attribution to typical phase-transformation of the films under ablation conditions is questionable.

A high sensitivity of the ablation observables on mode of film deposition is observed, which indicates that energy distribution during ablation is highly sensitive to the "structure" of the substrates. On the other hand, the examination of the desorbate $E_{\text {TOTAL }}$ indicates that a strikingly high percentage of the absorbed energy is deposited on the desorbates. Energy transfer from the deeper layers of the film to the ejecta appears to be highly efficient. This possibility is further 
demonstrated by the observation of high $E_{\text {trans }}$ and sputtering yields for $\mathrm{C}_{6} \mathrm{H}_{12}$ condensed on thick $\mathrm{C}_{6} \mathrm{H}_{5} \mathrm{Cl}$ films.

Two models are discussed in relationship to these observations, namely that of collisional sequences (Thomson, Johnson et al.) and a thermal model complemented with the assumption of film imperfections introducing barriers (bottlenecks") to the free distribution of the energy in the film. These models seem, at least qualitatively, to be able to account for most observations on the ablation of cryogenic films in the UV.

\section{Acknowledgements}

The work was supported by the Large Installations Plan DGXII (Project G/89100086/GEP) and by the Human Capital and Mobility Programme (No. ERBCHRXCT930157).

\section{References}

[1] a) Domen, K. and Chuang, T. J. (1989). J. Chem. Phys., 90, 3318; b) Domen, K. and Chuang, T. J. (1989). J. Chem. Phys., 90, 3332.

[2] Natzle, W. C., Padowitz, D. and Sibener, S. J. (1988). J. Chem. Phys., 88, 7975.

[3] Kutzner, J., Lindeke, G., Welge, K. H. and Feldman, D. (1989). J. Chem. Phys., 90, 548.

[4] a) Cousins, L. M. and Leone, S. R. (1989). Chem. Phys. Lett., 155, 162; b) Cousins, L. M., Levis, R. J. and Leone, S. R. (1989). J. Phys. Chem., 93, 5325; c) Cousins, L. M., Levis, R. J. and Leone, S. R. (1989). J. Chem. Phys., 91, 5731.

[5] Campos, F. X., Waltman, C. J. and Leone, S. R. (1993). Chem. Phys. Lett., 201, 399.

[6] a) Machini, M. and Hess, P. (1981). Chem. Phys. Lett., 77, 541; b) Schafer, B. and Hess, P. (1984). Chem. Phys. Lett., 105, 563; c) Braun, R. and Hess, P. (1993). J. Chem. Phys., 99, 8330.

[7] (a) Tsuboi, Y., Fukumura, H. and Masuhara, H. (1995). Appl. Phys. Let., 64, 2745;

(b) Tsuboi, Y., Hatanaka, K., Fukumura, H. and Masuhara, H. (1994). J. Phys. Chem., 98, 11237.

[8] (a) Georgiou, S., Syrrou, M., Kontoleta, P. and Koubenakis, A. "Photofragmentation and Reactivity Patterns in the Ablation of van der Waals Films. I: Ablation of Chlorobenzene Films at 248 nm", submitted to J. Chem. Phys; (b) Georgiou, S., Kontoleta, P., Syrrou, M. and Koubenakis, A. "Ablation of Mixed Chlorobenzene and Cyclohexane Films", to be submitted; (c) Georgiou, S., Koubenakis, A., Kontoleta, P. and Syrrou, M. (1996). "Induction and Memory Effects in the UVAblation of Weakly Absorbing van der Waals Films" (1996) Chem. Phys. Lett., 260, 166.

[9] Robin, M. B. (1974). Higher Excited States of Polyatomic Molecules (Academic Press, New York) vol II, pp. 209-237.

[10] Handbook of Chemistry and Physics, 61 ed., edited by R. C. Weast, and M. J. Astle (1980). (CRC, Boca Raton, FL).

[11] Braun, R. and Hess, P. (1993). Int. J. Mass Spectrosc. Ion Processes, 125, 229. 
[12] Sverdlova, O. V. (1959). Optics and Spectrosc., 6, 223.

[13] Brith, M., Lubart, R. and Steinberger, I. T. (1971). J. Chem. Phys., 54, 5104.

[14] Dabiri, A. E., Lee, T. J. and Stickney, R. E. (1971). Surf. Sci., 26, 522.

[15] Domalski, E. S. and Hearing, E. D. (1996). J. Phys. Chem. Refer. Data, 25, 171-172; pp. 231-234; pp. 175-178.

[16] Inagaki, T. (1972). J. Chem. Phys., 57, 2526.

[17] Sowers, B. L., Arakawa, E. T. and Birkhoff, R. D. (1971). J. Chem. Phys., 54, 2319.

[18] Berry, R. S., Rice, S. A. and Ross, J. (1980). Physical Chemistry II. Matter in Equilibrium: Statistical Mechanics and Thermodynamics (John Wiley: New York). pp. 836-854; 945-963.

[19] Zallen, R. (1983). The Physics of Amorphous Solids, Wiley: New York.

[20] Meyer, B. (1971). Low-Temperature Spectroscopy (American Elsevier Co., New York) and references therein.

[21] Hannay, N. B. (1976). Treatise on Solid State Chemistry, 3, Crystalline and Noncrystalline Solids, Plenum Press: New York.

[22] (a) Fraenkel, R. and Haas, Y. (1994). Chem. Phys., 186, 185; (b) Fraenkel, R. and Hass, Y. (1994). J. Chem. Phys., 100, 4324.

[23] Chemistry and Physics of Matrix-Isolation Species, edited by Andrews, L. and Moskovits, M. (North-Holland, Amsterdam, 1989).

[24] Westman, H., Demirev, P., Huth-Fehre, T., Bielawski, J. and Sundqvist, B. U. R. (1994). Int. J. Mass Spectrom. and Ion Processes, 130, 107.

[25] Thompson, M. W. (1968). Phil. Mag., 18, 377.

[26] Sigmund, P. (1969). Phys. Rev., 184, 383.

[27] (a) Garrison, B. J. and Johnson, R. E. (1984). Surf. Sci., 148, 388; (b) Cui, S., Johnson, R. E. and Cummings, P. T. (1988). Surf. Sci., 207, 186; (c) Cui, S., Cummings, P. T. and Johnson, R. E. (1989). Surf. Sci., 222, 491.

[28] Owrutsky, J. C., Raftery, D. and Hochstrasser, R. M. (1994). Ann. Rev. Phys. Chem., 45, 519 and references therein.

[29] Bondybey, V. E. and Brus, L. E. (1980). Adv. Chem. Phys., 41, 269 and references therein.

[30] (a) Sedlacek, A. J., Mansuento, E. S. and Wight, C. A. (1987). J. Am. Chem. Soc., 109, 6223; (b) Sedlacek, A. J. and Wight, C. A. (1988). Laser Chem., 9, 155.

[31] (a) Vertes, A., Gijbels, R. and Levine, R. D. (1990). Rapid Commun. Mass Spectrom., 4, 228; (b) Vertes, A. and Levine, R. D. (1990). Chem. Phys. Lett., 171, 284.

[32] Vertes, A. and Gijbels, R. (1993). Laser Ionization Mass Analysis, ed. A. Vertes, R. Gijbels and F. Adams (John Wiley: New York) chapter 3, and references therein.

[33] Levis, R. J. (1994). Ann. Rev. Phys. Chem., 45, 483 and references therein.

[34] Ishii, K., Nakayama, H., Yagasaki, Y., Ando, K. and Kawahara, K. (1994). Chem. Phys. Lett., 222, 117.

[35] Knozinger, E., Schuller, W. and Langel, W. (1993). J. Phys. Chem., 97, 927.

[36] Johnson, R. E. and Sundqvist, B. U. R. (1991). Rapid Commun. Mass Spectrom., 5, 574.

[37] Johnson, R. E. (1994). Int. J. Mass Spectrom. Ion Processes, 139, 574 and references therein.

[38] Heyd, D. V., Jensen, E. T. and Polanyi, J. C. (1995). J. Phys. Chem., 103, 461.

[39] Franck, J. and Rabinowitch, E. (1934). Trans. Faraday Soc., 30, 120.

[40] Pimentel, G. C. (1960). Radical Formation and Trapping in the Solid Phase in Formation and Trapping of Free Radicals, ed. A. Bass and H. Broida (Academic Press, Inc., New York, N.Y.) 